\title{
Hirschsprung's disease: a review of the morphology and physiology
}

\author{
E. R. Howard \\ M.S., F.R.C.S.
}

King's College Hospital, London, S.E.5*

MANY theories have been postulated to account for 'congenital megacolon' since the classical description by Hirschsprung in 1888 . At first the dilated bowel of the megacolon attracted most of the attention but later most features of the disease were attributed to the absence of ganglion cells in an undilated segment of distal bowel. More recently, however, morphological and physiological observations have suggested that the cause is a more complex abnormality of innervation which involves not only the ganglion cells but also the distribution of adrenergic and cholinergic nerves within the bowel wall. Variations in this abnormal innervation may account for differences in the clinical progress and presentation of patients with Hirschsprung's disease.

\section{Morphology}

Osler (1893) was probably the first to suggest that 'congenital megacolon' was caused by a defect in the innervation of the colon which resulted in a failure of normal evacuation. Three early reports of histological abnormalities in the Auerbach plexus of bowel distal to the megacolon (Tittel, 1901; Dalla Valle, 1920; Cameron, 1928) were mostly ignored, although all three suggested that the observations might indicate that there was a segment of bowel incapable of normal peristalsis.

A constant histological abnormality in Hirschsprung's disease was defined later in three papers which reported an absence of ganglion cells in variable lengths of rectum and colon, the aganglionosis always beginning in the lower part of the rectum and extending proximally (Whitehouse \& Kernohan, 1948; Zuelzer \& Wilson, 1948; Bodian, Stephens \& Ward, 1949). Large nerve trunks were found between the longitudinal and circular muscle (Figs. 1 and 2).

Early histochemical studies of aganglionic bowel showed that the large nerve trunks were strongly positive for acetylcholinesterase (AChE) and they were therefore regarded as cholinergic or parasympathetic nerves (Kamijo, Hiatt \& Koelle, 1953; Adams, Marples \& Trounce, 1960). Further work

\footnotetext{
* Present address: Medical University of South Carolina, Charleston, South Carolina, 29401, U.S.A.
}

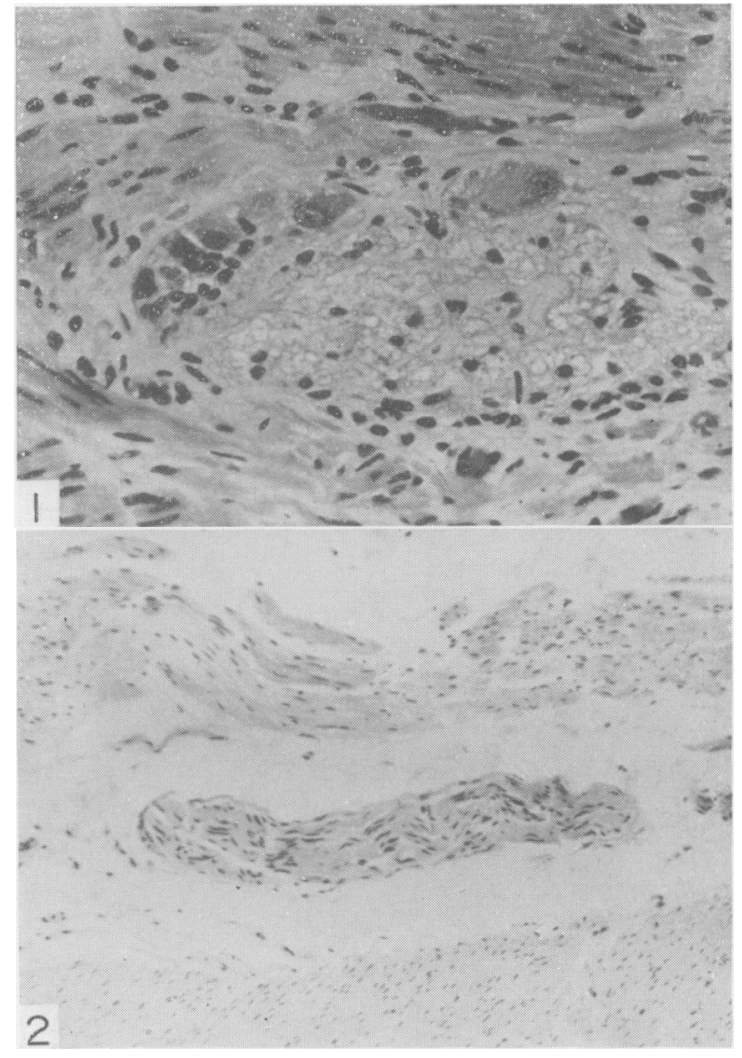

FIG. 1. A ganglion of Auerbach's plexus in normal bowel. Stained with $\mathrm{H} \& \mathrm{E}, \times 225$.

FIG. 2. Rectum from a patient with Hirschsprung's disease. Ganglia are absent but large nerve trunks are seen between the longitudinal and circular muscle. $\mathbf{H}$ $\& \mathrm{E}, \times 90$.

demonstrated AChE-positive nerves within the circular muscle (Niemi, Kouvalainen \& Hjelt, 1961 ; Meier-Ruge, 1968) and this suggested that the aganglionic bowel might be innervated by cholinergic fibres.

Recently the muscle innervation of Hirschsprung bowel has been re-investigated. Cholinergic nerves have been visualized by staining for acetyl 
cholinesterase (Garrett, Howard \& Nixon, 1969) whilst adrenergic nerves have been identified by a catecholamine fluorescence technique (Bennett, Garrett \& Howard, 1968; Garrett et al., 1969; Gannon, Noblett \& Burnstock, 1969).

Electron microscopy has been used to determine the normality or otherwise of the constituent axons of nerves revealed by histochemistry and to determine the presence or absence of neuro-effector or innervation sites within the muscle layers (Howard \& Garrett, 1970). If nerves within the muscle layers have a functional role then neuro-effector sites should be readily identifiable.

In normal colon and rectum the circular muscle contains moderate numbers of AChE-positive nerves, whilst the longitudinal muscle contains rather fewer (Fig. 3). The ganglia of the intermyenteric zone contain acetylcholinesterase but there is considerable variation in the staining of individual neurones.

In contrast, the greater proportion of fluorescent adrenergic nerves in normal bowel are found in close association with ganglia (Fig. 4) and only a few are present within the muscle layers themselves (Nor-

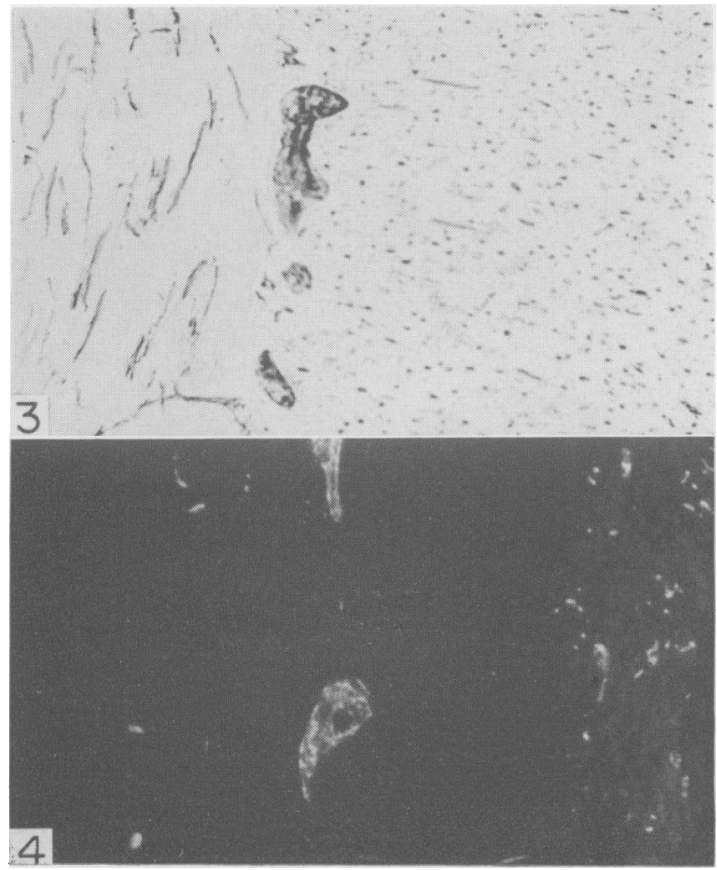

FIG. 3. Normal rectum stained for acetylcholinesterase. AChE-positive nerves are seen within both muscle layers. Ganglia show variation in staining. $\times 69$. NB-In Figs. 3, 4, 6, 7 and 8 the longitudinal muscle is on the left.

FIG. 4. Formalin induced fluorescence in normal rectum. Fluorescent adrenergic nerves are distributed mainly around ganglia. Few are seen within the muscle layers. $\times 63$. berg, 1964; Jacobowitz, 1965; Baumgarten, 1967). The adrenergic nerves are distributed mainly around the periphery of ganglia but the ganglion cells are never fluorescent and are therefore non-adrenergic. As with AChE-positive nerves the pattern of distribution of adrenergic fibres shows little variation at different levels of human colon and rectum.

With electron microscopy large numbers of nonmyelinated nerves are seen within the muscle layers of normal bowel arranged in typical Schwann-axon bundles. Each bundle may contain up to twenty axons per Schwann cell. The axons are often found partially bared of Schwann-cell cytoplasm in close proximity to muscle cells, the gaps being less than $500 \AA$. These arrangements are typical of neuroeffector or innervation sites within smooth muscle (Fig. 5).

Histochemical examination of bowel resected from patients with Hirschsprung's disease shows that in contrast to normal bowel there is a variation in the distribution of AChE-positive nerves at different levels of each specimen. There is also a variation in the numbers of nerves between different cases. Thus, the circular muscle of the distal rectum tends to contain more AChE-positive nerves than normal rectum at the same level (Fig. 6). Ascending the bowel these nerves gradually diminish in number until the most proximal aganglionic tissue contain fewer than normal. In addition, the zone above the aganglionic bowel where ganglia first appear, alse contains fewer muscular nerves than normal (Fig. 8). These observations confirm earlier work on the acetylcholinesterase content of Hirschsprung bowel by Kamijo et al. (1953). These authors used a manometric method of estimation and reported that there

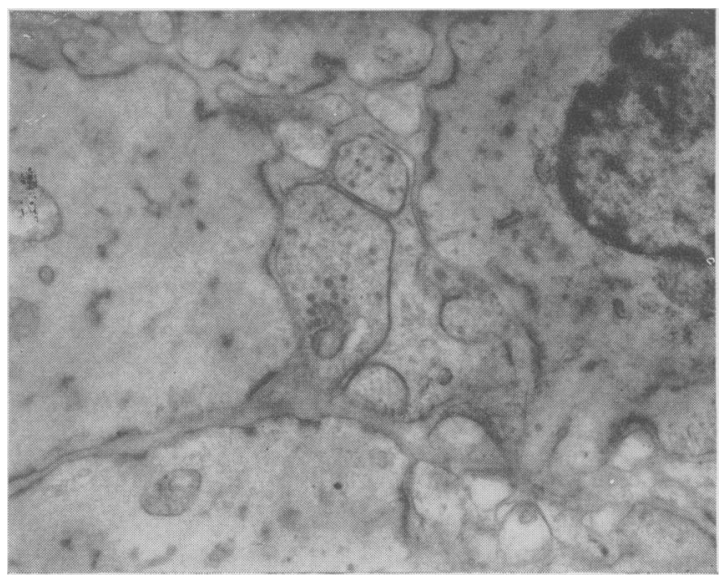

Fig. 5. Electron micrograph showing a vesiculated axon in close proximity to a circular muscle cell-an arrangement typical of smooth muscle innervation sites. $\times 10,000$. 
was much less acetylcholinesterase activity in proximal ganglionic tissue than in distal aganglionic bowel, and also that there was less than in normal controls. Further, recent pharmacological estimations of acetylcholine release from Hirschsprung bowel have shown that there are greater quantities of the transmitter in the distal contracted segment than in the proximal dilated bowel (Crema, 1971) and provide further confirmation of the pattern of cholinergic nerve distribution revealed by histochemistry.

The normal arrangement of adrenergic nerves is not possible in aganglionic bowel. Instead, the nerves are distributed through both muscle layers (Fig. 7). As with the AChE-positive nerves, the largest number of adrenergic fibres is found in the distal rectum and these numbers gradually decrease in a cephalad direction (Garrett et al., 1969; Gannon et al., 1969). As soon as ganglia appear in the sections the normal periganglionic arrangements are seen. Again there is variation in numbers of nerves between different cases, varying from many more than normal in some, to very few in others. It is of interest that in some specimens it is the circular muscle that is most heavily innervated with adrenergic nerves whilst in others it is the longitudinal muscle.

Electron microscopy of the large nerve trunks of the intermuscular zone of aganglionic bowel reveals the presence of a few myelinated axons amongst

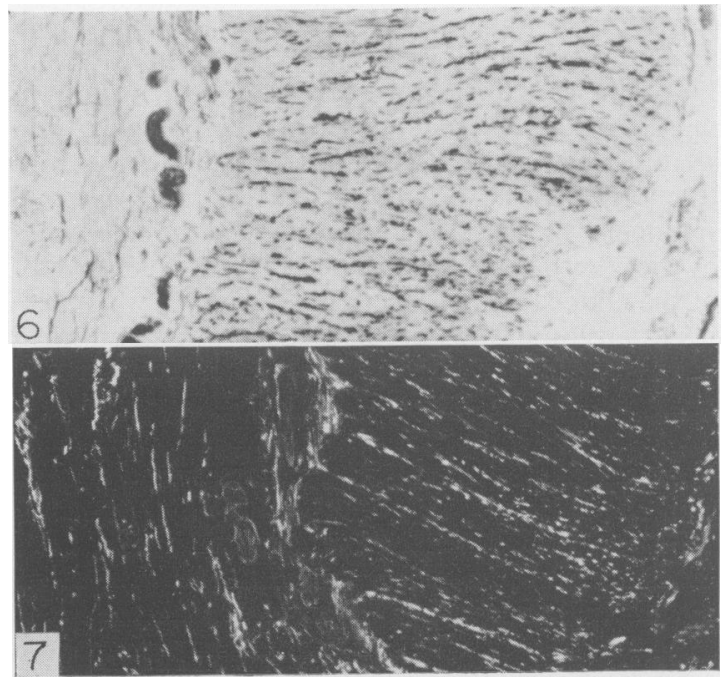

Fig. 6. Distal aganglionic bowel in Hirschsprung's disease stained for AChE. Densely stained nerve trunks are present in the intermyenteric zone. Many AChEpositive nerves are seen in the circular muscle. $\times 38$. Fig. 7. Formalin-induced fluorescence in aganglionic bowel. Large numbers of adrenergic nerves are seen within both longitudinal and circular muscle. $\times 40$.

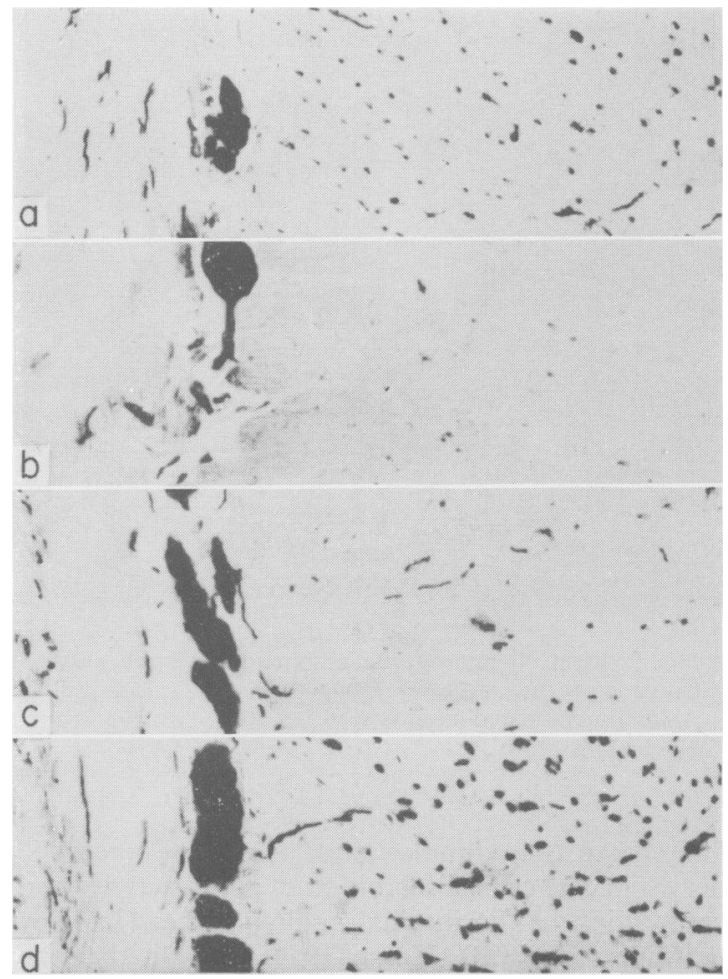

FIG. 8. Segments of bowel from different levels in the same case of Hirschsprung's disease stained for AChE activity. (a) Proximal ganglionic zone showing a ganglion and normal number of nerves in muscle layers. $\times 80$. (b) Distal ganglionic zone showing a ganglion but few nerves in the muscle. $\times 80$. (c) Proximal aganglionic zone showing nerve trunks but few nerves in the muscle. $\times 80$. (d) Distal aganglionic zone showing nerve trunks and many nerves in the circular muscle. $\times 80$.

hundreds of non-myelinated axons, the whole being surrounded by a sheath of perineural cells and collagen (Fig. 9). The ultrastructure of the non-myelinated axons within the muscle layers shows no obvious difference from normal except perhaps an increase in the numbers of axons in many of the Schwannaxon bundles. Sites similar to the neuro-effector junctions of normal bowel are easily identifiable, especially in distal tissue, and this suggests that the nerves are truly functional.

\section{Physiology}

Early theories on the pathophysiology of the functional obstruction in Hirschsprung's disease were often derived from experiments on the autonomic nervous system in animals. It was suggested, for example, that the obstruction might be due to overactivity of the sympathetic nervous system (Alvarez, 1922; Hunter, 1924) after experiments 


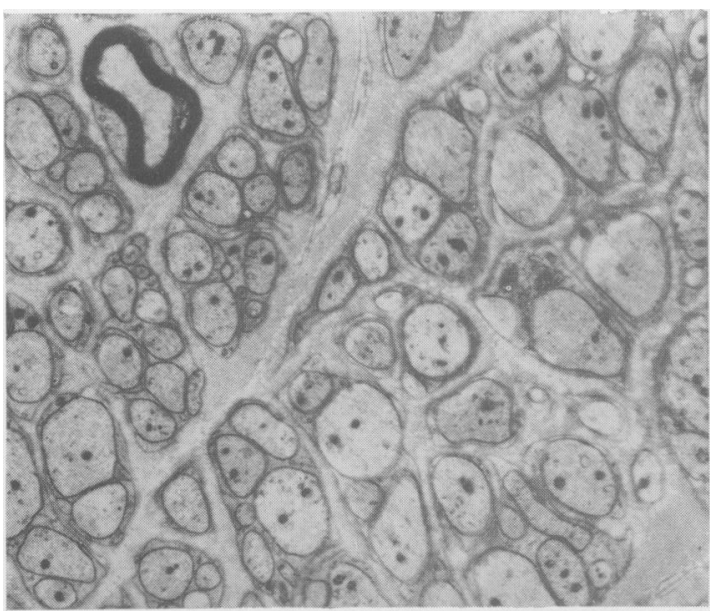

FIG. 9. Electron micrograph of portion of large nerve trunk from intermyenteric zone of aganglionic bowel. A small myelinated axon is seen. $\times 5600$.

had shown that stimulation of lumbar sympathetic nerves in cats caused inhibition of colon activity and contraction of the internal anal sphincter (Langley \& Anderson, 1895). Hurst (1934) thought that the disease was caused by a failure of relaxation of the internal sphincter and believed that it was analogous to achalasia of the cardia.

The first physiological studies of patients with Hirschsprung's disease were reported by Swenson, Rheinlander \& Diamond (1949) and Hiatt (1951). Recordings from small balloons which had been inserted to different levels of colon and rectum showed that contractions occurred in aganglionic segments but that this activity bore no relation to the peristaltic waves of the more proximal ganglionic bowel. These unco-ordinated movements of the aganglionic bowel were also seen on barium examinations.

Recently intraluminal pressure measurements have been used to study muscle activity in the rectum and anal sphincters of patients with Hirschsprung's disease. Synchronous measurements of activity at different levels were recorded with either rubbercovered probes carrying multiple small air-filled chambers (Lawson \& Nixon, 1967) (Fig. 10), or with larger balloons mounted in tandem (Schnaufer et al., 1967). Contractions of voluntary muscle in the external anal sphincter were monitored from electromyograph recordings taken with either needle or surface electrodes.

Recordings from the normal unstimulated rectum showed occasional co-ordinated contractions which produced only small rises of intraluminal pressure. Contractions of the muscle of the anal sphincter

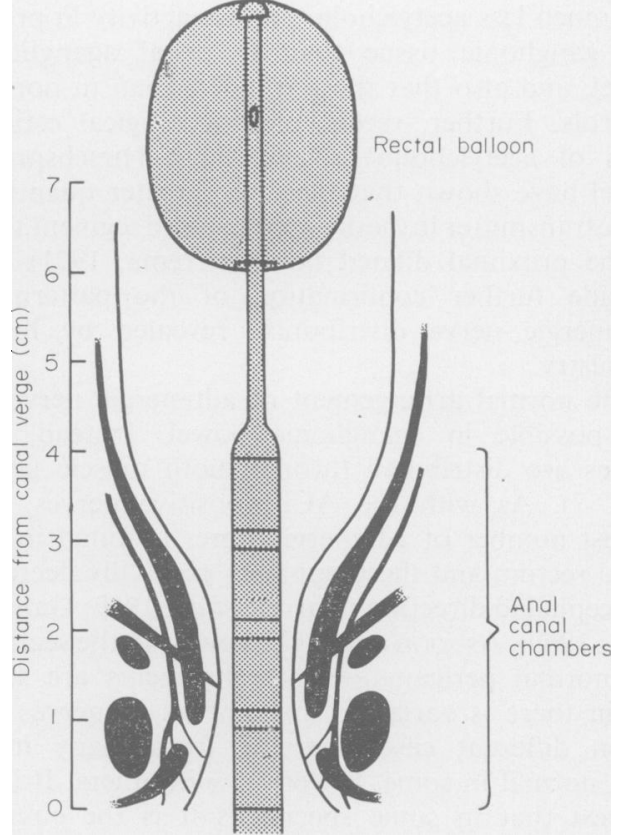

Fig. 10. Diagram of probe used to record intraluminal pressures in the anorectum.

could be recognized by two types of activity recordef from within the anal canal (Fig. 11). The smoot muscle of the internal sphincter produced rhythmical contractions with a frequency of $12-24 / \mathrm{min}$ and a mean pressure of $40-50 \mathrm{~cm} \mathrm{H}_{2} \mathrm{O}$ (Howard \& Nixon, 1968). In constrast, contractions of the voluntary muscle of the external sphincter were irregular and were accompanied by changes in the EMG. Contractions caused by stimulation of the external sphincter would usually mask the rhythmic activity of the internal sphincter especially in the lower half of the anal canal.

Distension of the normal rectum with an air-filled balloon was followed by a simple co-ordinated rectal contraction of $15-20 \mathrm{sec}$ duration. The rectum then adapted to the distending volume with the result that increasing increments of air produced only a gradual rise in rectal pressure.

The effects of rectal distension on normal anal canal pressures were complex as they depended on the different reflex responses of the internal and external sphincters (Fig. 11). A fall in pressure and inhibition of rhythmical activity similar in duration to the rectal contraction were recorded from the smooth muscle of the internal sphincter. In contrast the activity of the voluntary muscle of the external sphincter was increased by rectal distension so that when continence was threatened by the injection of a large quantity of air the consequent rise in external 
O.C. $10 \frac{1}{2}$ years

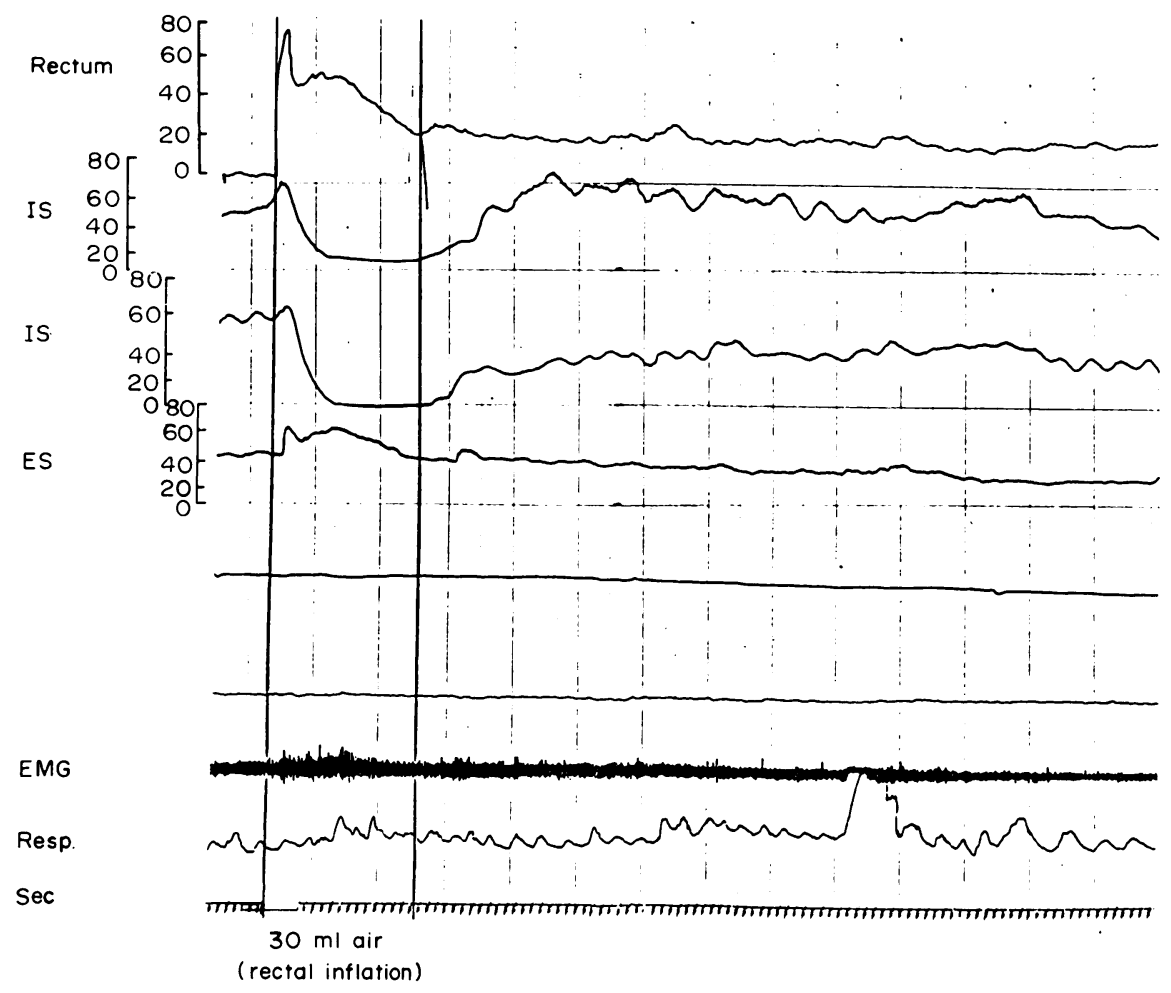

FIG. 11. Normal anal sphincter response to rectal distension. A rectal contraction is accompanied by inhibition of the internal sphincter (IS) and contraction of the external sphincter (ES). The pressures are in $\mathrm{cm}$ of water.

sphincter pressure would more than compensate for the relaxation of the internal sphincter.

Recordings taken from the aganglionic segments of rectum and anal canals of patients with Hirschsprung's disease differeci markedly from the normal controls (Fig. 12). Whereas normal rectum adapted to a distending volume of air, aganglionic bowel produced a more sustained rise of intraluminal pressure which was modified to some extent by contractions which varied in duration and strength. Further, recordings from the anal canal revealed a complete absence of reflex inhibition in the internal

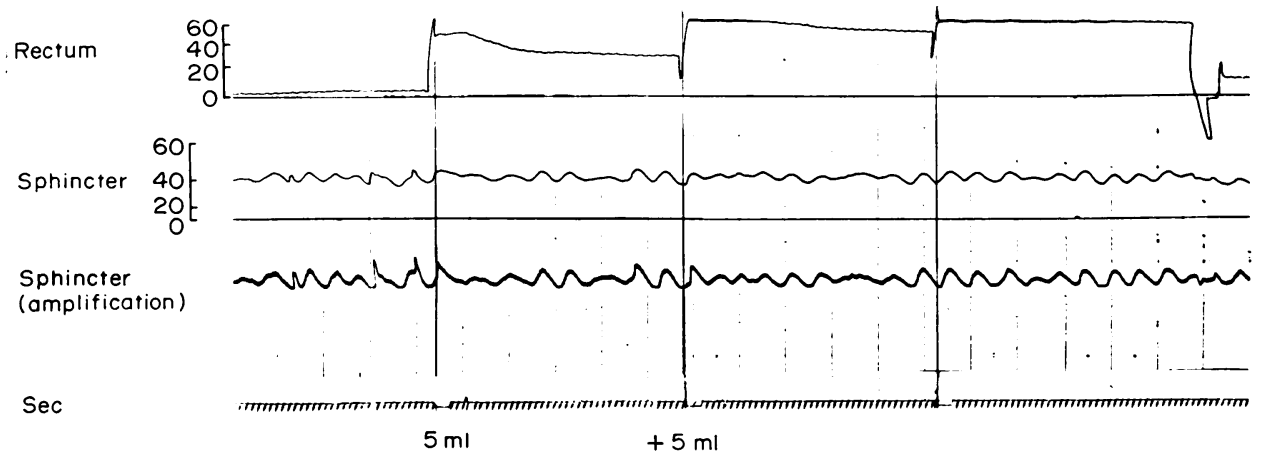

FIG. 12. Hirschsprung's disease in an infant of 5 weeks. Rectal distension has no effect on the muscle activity of the internal sphincter. 
sphincter. Rhythmical contractions of this muscle were rather more prominent than in normal controls and were not reduced by rectal distension. No differences were detected in the responses of the external anal sphincter.

In summary, there are three abnormalities of muscle activity in aganglionic bowel which can be recorded with intraluminal balloon techniques.

(1) There is an absence of co-ordinated peristalsis.

(2) Unco-ordinated contractions of variable strength and duration replace normal motility.

(3) Inhibitory reflexes between rectum and internal anal sphincter are absent.

\section{Discussion}

In normal bowel the motility of the hind-gut is controlled by the cholinergic parasympathetic nerves of the sacral outflow and the adrenergic nerves of the lumbar sympathetic system. The cholinergic nerves are motor in function whilst the adrenergic system is inhibitory. Myenteric ganglion cells coordinate motor activity and almost certainly play a part in inhibitory reflexes through their close relationship with adrenergic nerves.

The bowel dysfunction in Hirschsprung's disease is the result of a complex abnormality which involves all parts of this autonomic innervation. Myenteric ganglion cells are absent in a variable length of distal bowel and consequently peristalsis and the normal forward propulsion of bowel contents does not occur. The resulting obstruction is probably augmented by the abnormalities of cholinergic and adrenergic innervation. Cholinergic nerves are present in aganglionic bowel, often in very large numbers, and their functional role is indicated by the presence of neuro-effector sites within the muscle layers. Adrenergic nerves are also present but their normal arrangement around ganglion cells is not possible. The unco-ordinated motor effects of the cholinergic nerves and the absence of normal adrenergic inhibitory mechanisms could both accentuate the functional obstruction caused by the absence of peristalsis. Paralysis of cholinergic nerves may also explain the observation that relaxation of aganglionic bowel may be induced by spinal anaesthesia (Ehrenpreis, 1946; Bodian et al., 1949).

The demonstration of fewer muscular nerves than normal in the ganglionic bowel adjacent to the aganglionic segment may be a further factor in Hirschsprung's disease. The sparse distribution of cholinergic nerves in this area suggests that it may be a zone of reduced motor activity the effect of which would be to accentuate any obstruction caused by unco-ordinated motor activity in more distal bowel.

It has been suggested that variation in innervation may explain the variation in clinical presentation. Although the majority of patients have some delay in the passage of meconium during the first few days of life only a proportion go on to complete intestinal obstruction. A few appear to develop a satisfactory $\frac{a}{c}$ bowel habit for a time and present later with constipation whilst others suffer repeated attacks of subacute obstruction.

The length of the aganglionic segment is an important factor and where this extends proximal to the sigmoid colon it is very likely to produce intestinal obstruction in the neonatal period. It is well known, however, that even in disease restricted to the rectosigmoid the degree of obstruction may vary from case to case. Recently some correlation has been found between the severity of clinical presentation and the numbers of cholinergic nerves within the circular muscle of distal aganglionic bowel. Aganglionic segments containing large numbers of nerves have been found in patients presenting with complete obstruction in the neonatal period (Garrett et al., 1969), whilst segments with fewer cholinergic nerves have been resected from children who presented later in life with lesser degrees of obstruction.

The degree of cholinergic innervation and consequent motor activity in aganglionic segments may therefore explain some of the variation in clinical presentation whilst further variability may be a result of diminished cholinergic nerve supply in the dista ganglionic bowel, or transitional zone.

In summary, recent work suggests that the funeo tional obstruction of Hirschsprung's disease is due to at least four abnormalities in the innervation of the affected segment of bowel:

(1) An absence of ganglion cells with a consequent lack of co-ordinated peristalsis.

(2) An absence of normal sympathetic innervation preventing effective relaxation.

(3) Increased numbers of cholinergic nerves in distal bowel inducing unco-ordinated motor activity.

(4) Reduced cholinergic innervation of more proximal areas producing a zone of poor motor activity.

The management of patients with Hirschsprung's disease has recently been reviewed in detail by Nixon (1971) who included a description of the operations used for the resection of aganglionic bowel. The recent histochemical studies suggest that these resections should always include adequate lengths of the dilated ganglionic as well as the undilated aganglionic bowel to include the zone with a reduced muscle innervation. It is possible that inadequate removal of this tissue may lead to the incomplete relief of symptoms.

The histochemical assessment of innervation has been used for the diagnosis of Hirschsprung's disease on rectal biopsy specimens. Meier-Ruge (1968) considered that an increased number of cholinesterasepositive nerves was diagnostic of the disease whilst 
Gannon et al. (1969) depended on the increased adrenergic innervation of the muscularis mucosae. The clear identification of ganglia with rapid non-specific esterase staining techniques has also been reported (Garrett \& Howard, 1969). An alternative technique for the preliminary diagnosis of congenital aganglionosis has been developed from the physiological studies. It has been shown that in the investigation of bowel dysfunction rectal biopsy can be restricted to patients in whom there is an absence of the normal recto-anal inhibitory reflex (Howard \& Nixon, 1968; Tobon et al., 1968) as this reflex is present in patients presenting with constipation from other causes.

\section{Acknowledgments}

I wish to thank Mr K. Davies for the preparation of the figures.

Figures 1 and 2 are reproduced by courtesy of J. \& A. Churchill Ltd, and Fig. 10 by courtesy of Archives of Disease in Childhood.

\section{References}

Adams, C.W.M., Marples, E.A. \& Trounce, J.R. (1960) Achalasia of the cardia and Hirschsprung's disease: The amount and distribution of cholinesterases. Clinical Science, 19, 473.

Alvarez, W.C. (1922) The Mechanics of the Digestive Tract. Paul B. Hoeber, New York.

BAUMGARTEN, H.G. (1967) Uber die verteilung von catecholaminen im darm des menschen. Zeitschrift für Zellforschung und Mikroskopische Anatomie, 83, 133.

Bennett, A., Garrett, J.R. \& Howard, E.R. (1968) Adrenergic myenteric nerves in Hirschsprung's disease. British Medical Journal, 1, 487.

Bodian, M., Stephens, F.D. \& WaRd, B.C.H. (1949) Hirschsprung's disease and idiopathic megacolon. Lancet, i, 6.

CAMERON, J.A.M. (1928) On the aetiology of Hirschsprung's disease. Archives of Disease in Childhood, 3, 210.

Crema, A. (1971) Personal communication.

Dalla Valle, A. (1920) A ricerche istologiche su di un caso di megacolon congenito. Pediatria (Napoli), 28, 740.

EHRENPREIS, T. (1946) Megacolon in the newborn. A clinical and roentgenological study with special regard to the pathogenesis. Acta chirurgica Scandinavica, 94, Suppl. 112.

Gannon, B.J., Burnstock, G., Noblett, H.R. \& CampBELL, P.E. (1969) Histochemical diagnosis in Hirschsprung's disease. Lancet, i, 894.

GanNon, B.J., Noblett, H.R. \& Burnstock, G. (1969) Adrenergic innervation of bowel in Hirschsprung's disease. British Medical Journal, 3, 338.

GarRetT, J.R. \& Howard, E.R. (1969) Histochemistry and the pathology of Hirschsprung's disease. Proceedings of the Royal Microscopical Society, 4, 76.

Garrett, J.R., Howard, E.R. \& Nixon, H.H. (1969) Autonomic nerves in rectum and colon in Hirschsprung's disease. Archives of Disease in Childhood, 44, 406.

HiatT, R.B. (1951) The pathologic physiology of congenital megacolon. Annals of Surgery, 133, 313.
HiRsChSPRUNG, H. (1888) Stuhlträgheit neugeborener in folge von dilatation und hypertrophie des colons. Jahresbericht Kinderheilk, 27, 1.

Howard, E.R. (1970) Hirschsprung's disease: A physiological and morphological study. M.S. thesis, London.

HowaRD, E.R. \& GaRRETT, J.R. (1970) Electronmicroscopy of myenteric nerves in Hirschsprung's disease and in normal bowel. Gut, 11, 1007.

Howard, E.R. \& Nixon, H.H. (1968) The internal anal sphincter: Observations on the development and mechanism of inhibitory responses in premature infants and children with Hirschsprung's disease. Archives of Disease in Childhood, 43, 569.

Hunter, J.M. (1924) The postural influence of the sympathetic innervation of voluntary muscle. Medical Journal of Australia, 1, 86.

Hurst, A. (1934) Anal achalasia and megacolon. Guy's Hospital Reports, 84, 317.

JACOBOWITZ, D. (1965) Histochemical studies of the autonomic innervation of the gut. Journal of Pharmacology and Experimental Therapeutics, 149, 358.

Kamijo, K., Hiatt, R.B. \& Koelle, G.B. (1953) Congenital megacolon: A comparison of the spastic and hypertrophied segments with respect to cholinesterase activities and sensitivities to acetylcholine, DFP, and the barium ion. Gastroenterology, 24, 173.

LANGLey, J.N. \& ANDERSON, H.K. (1895) On the innervation of the pelvic and adjoining viscera. 1: The lower portion of the intestine. Journal of Physiology, 18, 67.

Lawson, J.O.N. \& Nixon, H.H. (1967) Anal canal pressures in the diagnosis of Hirschsprung's disease. Journal of Pediatric Surgery, 2, 544.

Meier-Ruge, W. (1968) Das megacolon. Seine diagnose und pathophysiologie. Virchows Archiv: Abteilung A: Pathologische Anatomie, 344, 67.

Niemi, M., Kouvalainen, K., \& Huelt L. (1961) Cholinesterase and monoamine oxidase in congenital megacolon. Journal of Pathology and Bacteriology, 82, 363.

NixON, H.H. (1971) Hirschsprung's disease. British Journal of Hospital Medicine, 5, 199.

NorberG, K.A. (1964) Adrenergic innervation of the intestinal wall studied by fluorescence microscopy. International Journal of Neuropharmacology, 3, 379.

OSLER, W. (1893) Cases of dilatation of the colon in young children. Bulletin of the Johns Hopkins Hospital, 4, 41.

Schnaufer, L., Talbert, J.L., Haller, J.A., Reid, N.C.R.W., Tobon, F. \& Schuster, M.M. (1967) Differential sphincteric studies in the diagnosis of ano-rectal disorders of childhood. Journal of Pediatric Surgery, 2, 538.

Swenson, O., Rheinlander, H.F. \& Diamond, I. (1949) Hirschsprung's disease: A new concept of the aetiology. New England Journal of Medicine, 241, 551.

TitTEL, K. (1901) Ueber eine angeborene missbildung des dickdarmes. Wiener Klinische Wochenschrift, 14, 903.

Tobon, F., Reid, N.C.R.W., Talbert, J.L. \& Schuster, M.M. (1968) Non-surgical test for the diagnosis of Hirschsprung's disease. New England Journal of Medicine, 278, 188.

Whitehouse, F.R. \& Kernohan, J.W. (1948) Myenteric plexus in congenital megacolon. Archives of Internal Medicine, 82, 75 .

Zuelzer, W.W. \& Wilson, J.L. (1948) Functional intestinal obstruction on a congenital neurogenic basis in infancy. American Journal of Diseases of Children, 75, 40. 